\title{
Construire un regard sociohydrologique (3). Expérimenter un canevas pour engager la négociation interdisciplinaire
}

\author{
Anne-Laure Collard ${ }^{1, *}$, Jeanne Riaux ${ }^{2}$, Sylvain Massuel $^{3}$, Bruno Bonté $^{4}$, Martin Laurenceau ${ }^{5}$, \\ Bastien Richard ${ }^{6}$ et Anne Rivière-Honegger ${ }^{7}$ \\ ${ }^{1}$ Sociologie, INRAE, UMR G-Eau, Montpellier, France \\ 2 Anthropologie, IRD, UMR G-Eau, Montpellier, France \\ 3 Hydrogéologie, IRD, UMR G-Eau, Montpellier, France \\ 4 Modélisation et simulation sociale, INRAE, UMR G-Eau, Montpellier, France \\ 5 Sciences de gestion de l'environnement, AgroParisTech, UMR G-Eau, Montpellier, France \\ 6 Agrohydrologie, AgroParisTech, UMR G-Eau, Montpellier, France \\ 7 Géographie, CNRS, UMR EVS, Lyon, France
}

\begin{abstract}
Résumé - Troisième volet d'un travail sur la démarche sociohydrologique, ce retour d'expérience s'intéresse aux modalités du dialogue interdisciplinaire en l'absence d'une expérience de terrain partagée. Un postulat a guidé la confection d'un «canevas» pour structurer les échanges au sein d'un groupe de chercheurs : la nécessité de partager des expériences pour négocier les points de convergence entre regards des sciences de la nature et de la société sur un même objet, ici les canaux d'irrigation communautaires. L'analyse du processus de construction, d'expérimentation et d'évaluation de ce canevas est entendue comme élément à part entière du processus interdisciplinaire. La double perspective sur laquelle repose cette contribution, expérimentation et réflexivité, permet un retour sur les modalités du dialogue interdisciplinaire et examine l'importance des frustrations dans la progression de la négociation.
\end{abstract}

Mots-clés : environnement / ressources naturelles / méthodologie / dialogue interdisciplinaire / réflexivité

\begin{abstract}
Development of a socio-hydrological approach (3). Testing a framework for interdisciplinary negotiation. This paper is the third part of a reflective work on building a sociohydrological approach. It focuses on the interdisciplinary dialogue between members of a research team associating the social and biophysical sciences. The key point is the construction of the interdisciplinary dialogue when it is not anchored in the previous experience of shared fieldwork. The creation of a framework to structure the dialogue is based on the premise that sharing experiences is crucial for negotiating a convergence between social and biophysical perspectives on reality. The first stage is to select an object on which to focus a collective analysis, in the present case the small-scale irrigation canals. The second stage consists in testing the framework during a three-day workshop. Third comes the collective negotiation of the analysis presented in the paper. Analysis of the construction, experimentation and evaluation process of the framework is considered an integral part of the interdisciplinary process. The dual approach of this contribution, i.e., experimentation and reflexivity, allows the analysis of the modalities of the interdisciplinary dialogue and questions the importance of frustrations in the progress of the interdisciplinary negotiation.
\end{abstract}

Keywords: environment / natural resources / methodology / interdisciplinary dialogue / reflexivity

Depuis les années 2000, l'interdisciplinarité fait parfois figure de "passage obligé » (Arnauld de Sartre et Petit, 2018) dans une nouvelle forme d'économie de la connaissance (Stengers, 2013). Pourtant, si l'histoire de

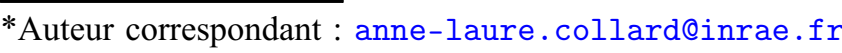

l'interdisciplinarité est déjà longue, le dialogue entre chercheurs des sciences de la nature et des sciences de la société ne peut s'appuyer sur une épistémologie stabilisée pour cadrer les échanges. Au contraire, l'interdisciplinarité «élargie» (Jollivet et Legay, 2005) progresse par l'expérience, en se confrontant à des 
écueils, en explorant des solutions. Dès lors, le retour d'expérience sur les pratiques interdisciplinaires est l'une des voies à privilégier pour construire cette épistémologie et favoriser une approche engagée de l'interdisciplinarité (Mathieu, 2012).

Ces retours sur la pratique interdisciplinaire reflètent les difficultés du dialogue, mettant en particulier l'accent sur l'absence de référentiels et de vocabulaire communs, le sentiment d'instrumentalisation, ou encore la lenteur de la construction d'une compréhension réciproque (Mathieu et al., 1997). Dans la continuité de retours d'expérience publiés dans la revue Natures Sciences Sociétés, des chercheurs de l'équipe SocioHydro ${ }^{1}$ ont proposé une série de témoignages réflexifs sur la construction d'un regard sociohydrologique. Les deux premiers articles identifiaient les archives et le terrain en commun comme catalyseurs de ce dialogue interdisciplinaire (Riaux, 2013; Riaux et Massuel, 2014). Ces témoignages reviennent sur la portée heuristique de l'interdisciplinarité et insistent sur l'importance d'un temps long d'échanges sur le terrain autour d'objets communs. Sans nier cette temporalité, nous avons cherché à interroger la manière d'engager le dialogue interdisciplinaire lorsque ces conditions de collaboration ne sont pas réunies. Et si commencer ce dialogue en amont d'une rencontre formalisée permettait de prendre la mesure du temps, de l'engagement et de la réflexivité qu'implique la pratique de l'interdisciplinarité et ainsi d'envisager préalablement au travail de terrain, les modalités de ce dialogue ? Ce troisième volet réflexif sur la construction «sociohydrologique» est focalisé sur les possibles modalités d'une telle construction du dialogue interdisciplinaire.

Dans la lignée des ateliers pratiques organisés par NSS pour faire valoir la portée heuristique de l'écriture (Pivot et Mathieu, 2007), nous avons cherché à expérimenter une méthode, à la vivre et à la faire vivre, afin d'engager le dialogue. Il s'agissait, suivant la recommandation de Jollivet et Legay (2005), d'accorder de l'importance à une réflexion méthodologique pour et dans la pratique de l'interdisciplinarité. Nous avons ainsi suivi leur idée d'esquisser un «canevas de départ» pour initier les débats à venir et les moduler par la suite. Ce «canevas» est envisagé comme un cadre, un point de départ non contraignant, dont l'ambition est de poser des premiers éléments de discussion pour enclencher le processus interdisciplinaire (Jollivet et Legay, 2005). À notre tour, nous avons donc construit une trame afin de cadrer un atelier qui a réuni une douzaine de chercheurs autour de canaux d'irrigation communautaires. Ce

\footnotetext{
${ }^{1}$ Cette équipe de l'UMR G-Eau rassemble des chercheurs dont l'objectif est de poser un regard interdisciplinaire sur les territoires de l'eau en se nourrissant du dialogue entre sciences hydrologiques et sciences de la société.
}

cadrage, qui fait l'objet de notre réflexion, s'inspire à la fois des ancrages disciplinaires des organisateurs ${ }^{2}$ et de leurs expériences préalables de l'interdisciplinarité. L'ambition du présent texte est d'analyser le processus d'élaboration, d'expérimentation et d'évaluation de ce «canevas de départ». Nous interrogeons les hypothèses qui le sous-tendent en associant une analyse de sa mise en œuvre lors de l'atelier et un travail réflexif mené $a$ posteriori et dont procède l'écriture même de cette contribution. Le travail d'écriture collective est alors considéré comme partie intégrante du processus de dialogue . $^{3}$.

\section{Tisser un canevas pour engager le dialogue}

La construction du canevas avait pour projet de façonner une expérience commune pour faire émerger des points de convergence entre participants et, dans le même temps, exprimer des divergences de points de vue issues des expériences individuelles. Convergences et divergences intégraient ainsi un travail de négociation autour du vécu et du regard de chacun dans le dispositif interdisciplinaire (Massuel et al., 2018). Pour cela, les organisateurs ont conçu un cadre articulé autour de l'objet hydraulique et des pratiques de terrain. En effet, le rapport des participants aux disciplines scientifiques, donc au dialogue interdisciplinaire, présentait une grande variété, certains se définissant comme «disciplinés», d'autres affirmant une posture mobilisant plusieurs disciplines. Cependant, tous travaillaient dans des situations géographiques diversifiées caractérisées par la présence de canaux d'irrigation communautaires. Dans ce contexte, l'ambition du canevas était à la fois de délimiter l'espace de dialogue tout en le rendant suffisamment ouvert pour permettre l'expression des positions individuelles.

\footnotetext{
${ }^{2}$ L'atelier s'est tenu dans la vallée de la Cèze (Cévennes), terrain du projet BEALS (Zone atelier Bassin du Rhône [ZABR], Agence de l'eau Rhône-Méditerranée-Corse). Organisé par A.-L.Collard (sociologue), F. Molle (géographe), A. Rivière-Honegger (géographe) et J. Riaux (anthropologue), il a réuni B. Bonté (modélisation et simulation sociale), C. Distinguin (agronome), S. Ghiotti (géographe), A. Hmouri (sociologue), N. Kherbache (économiste), M. Laurenceau (sciences de gestion de l'environnement), S. Massuel (hydrogéologue), C. Récalt (économiste), B. Richard (agrohydrologue) et T. Ruf (agrogéographe).

${ }^{3}$ La signature de ce texte reflète ce processus: les trois premiers auteurs sont les porteurs de la démarche sociohydrologique, par ordre d'implication dans l'écriture ; puis les participants ayant contribué à l'écriture sont listés dans l'ordre alphabétique.
} 


\section{Ancrer le dialogue autour d'un objet hydraulique}

Les objets techniques liés à l'eau représentent une opportunité pour saisir et interroger collectivement les relations qui se nouent entre environnement et sociétés. Les canaux communautaires sont des «objets hybrides" (Latour, 2013) au sens où ils ne sont ni seulement sociaux, ni seulement naturels ou techniques, mais traversés de ces multiples dimensions. L'étude de l'inscription matérielle de ces canaux permet ainsi de révéler des dynamiques sociales anciennes et récentes (Rivière-Honegger et Ruf, 2000 ; Ingold, 2008; Hmouri et al., 2017), tout autant que les effets de l'action humaine sur les régimes hydrologiques et sur les territoires de l'eau (Ghiotti, 2007). En outre, derrière l'apparente simplicité du canal, se dissimule une complexité qui peut conduire à des divergences d'interprétation. Néanmoins, l'objet hydraulique peut être saisi par tous en ce qu'il permet de poser un regard sur un élément descriptible. À ce titre, les canaux sont susceptibles de catalyser des intérêts a priori éloignés et de conduire à l'engagement du dialogue.

C'est la raison pour laquelle nous avons mis les canaux communautaires au cœur du canevas d'interaction, en proposant d'ancrer les discussions sur le cas concret des canaux anciens en Cévennes : les béals. Le cas cévenol est un terrain (ré)investi depuis 2018 dans le cadre d'un projet de recherche qui interroge la «modernisation ${ }^{4}$ » de ces ouvrages dont certains ont été érigés dès le XII ${ }^{\mathrm{e}}$ siècle pour l'alimentation de moulins et pour l'irrigation (Valat de Chapelain, 1983). Depuis 2008, les habitants de Génolhac sont confrontés aux mesures d'économie d'eau issues du Plan de gestion de la ressource en eau. Postulant que l'histoire en train de se faire, pourtant si « locale» et « ordinaire», peut receler une portée universelle (Wateau, 2016), les organisateurs de l'atelier ont voulu mettre au centre de l'expérience collective les réactions et les réponses matérielles des habitants à ces évolutions (Fig. 1). Le pari était alors de considérer les béals de Génolhac comme catalyseurs de convergences scientifiques.

\section{Délimiter un espace de dialogue ouvert}

Le canevas avait aussi pour fonction de délimiter l'espace du dialogue. Partant du fait que la question du devenir des canaux communautaires se pose au Nord comme au Sud, trois thèmes de réflexion ont été proposés au groupe. Le premier, "réinvention des canaux d'irrigation communautaire», invitait à interroger les

\footnotetext{
${ }^{4}$ La notion de modernisation renvoie à la volonté publique de rendre le béal plus efficient hydrauliquement et de restructurer les modalités d'organisation des associations d'usagers.
}

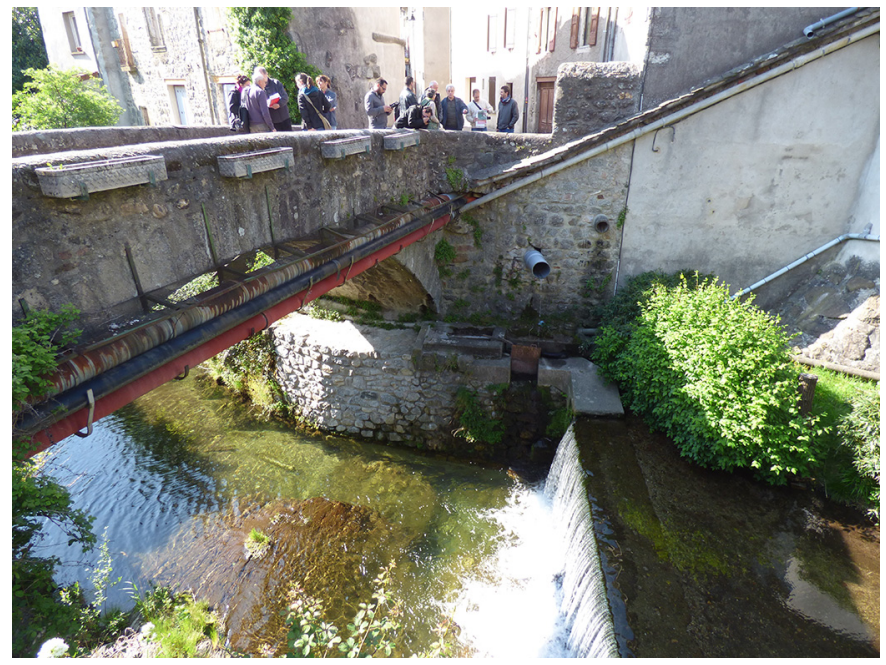

Fig. 1. Ouvrages hydrauliques à Génolhac, point de départ d'une matinée sur le terrain avec les responsables des associations d'usagers. Cliché: T. Ruf.

rapports des acteurs à l'objet technique. Le second, «production de connaissances et incertitudes sur les canaux ", amenait à discuter de l'articulation des formes de production des savoirs sur l'eau. Le troisième, «histoire sociohydraulique et interventions publiques», visait à aborder l'histoire longue des ouvrages à travers le dialogue entre administrations et communautés d'usagers.

Chacun de ces thèmes était pensé pour être nourri de questions transversales. Le parti pris était de favoriser un raisonnement par analogie, qui prendrait son sens empiriquement mais résonnerait ailleurs et autrement. Cette invitation à déplacer les regards reposait sur trois «glissements de terrain». Le premier concernait la rencontre des disciplines. Les participants étaient incités à réfléchir à l'influence de leur propre posture sur leurs observations et leurs productions de savoirs à propos des canaux. Le second visait le dépassement de la dichotomie Nord/Sud classiquement opérée. Le troisième interrogeait les temporalités dans la formulation et la construction des questions de recherche (Araújo et Guimarães, 2018).

L'idée initiale était celle d'une entrée collective sur le terrain, sans proposition de cadre théorique et/ou conceptuel fixé en amont. Mais, en réalité, le cadrage proposé reposait sur des soubassements théoriques implicites. Il n'était, par exemple, pas anodin d'introduire des dimensions historiques, politiques ou cognitives dans les axes de réflexion. Ces choix reflètent l'identité disciplinaire des organisateurs, tous relevant des sciences de la société, et leur lecture des problématiques liées aux canaux communautaires. Une réflexion cadrée par des chercheurs en sciences de la nature aurait certainement donné plus de place aux 
influences que les canaux d'irrigation peuvent avoir sur l'environnement et, en particulier, sur les débits des rivières.

\section{Une boîte à outils pour faciliter les négociations}

Dans le dialogue interdisciplinaire «élargi», l'un des principaux écueils à surmonter est l'absence de «culture disciplinaire » commune. Il n'y a pas d'évidences ou de références partagées sur lesquelles se reposer. L'expérience montre d'ailleurs qu'il vaut parfois mieux mettre de côté les quelques points considérés comme acquis. Le recours aux notions de «ressource», «systèmes» ou encore «dynamiques» est un bon exemple des malentendus qui peuvent s'immiscer dans le dialogue, tant leurs significations peuvent varier. Le canevas a donc été pensé dans l'idée de sortir des habitudes discursives de nos disciplines - ou indisciplines - respectives pour aborder la description, la problématisation et toute autre forme d'interprétation, de manière «neuve». Plusieurs outils ont été mobilisés pour cela.

L'observation et la réalisation d'entretiens ont donné forme au travail de terrain. Bien que le format court de l'atelier ne permette pas de mobiliser pleinement cette pratique dont la portée s'inscrit dans le temps long, la réflexion collective a été impulsée à partir d'un travail collectif de recueil de données qualitatives (Encadré 1).

Encadré 1. Déroulé du travail de terrain à Génolhac, Cévennes.

Le travail de terrain s'est déroulé sur une matinée. Les participants étaient répartis en trois groupes dont la composition avait été pensée au préalable, de manière à favoriser à la fois la rencontre de regards différents et un certain équilibre entre orientations disciplinaires. L'idée était d'éviter la prise d'ascendant d'une orientation (nature ou société) sur l'autre.

Chaque groupe a été accompagné par le responsable d'un canal, ce qui permettait aux participants de découvrir une variété de situations. Les groupes ont été guidés découvrant progressivement les modalités de gestion des canaux et leurs usages. Ils ont aussi abordé les difficultés rencontrées, notamment dans l'entretien des canaux et la mise en œuvre de la réglementation.

Lors de cette matinée, les participants avaient pour consigne de rassembler du «matériau ethnographique» (photographies, extraits d'entretiens, observations, etc.) pour alimenter les discussions à venir.

Des exercices de présentation et de mise en confiance inspirés de techniques de théâtre de l'opprimé ont été proposés ${ }^{5}$, dans l'idée de créer une arène d'échange suffisamment confortable pour que chacun accepte de sortir des routines de ses postures scientifiques. L'intérêt de cet emprunt était de déplacer le rôle des participants de l'atelier d' «invité » à celui d' «acteur» du dialogue. Il visait aussi à rompre une forme de hiérarchie intériorisée de la légitimité scientifique -implicite mais non moins présente - entre chercheurs dont la différence d'expérience, notamment, peut se traduire par des hésitations à s'exprimer (Bühler et al., 2006).

Un travail autour de la mise en images a également servi de support d'expression et de dialogue. L'usage du dessin s'inspirait de la proposition de Calandra (2013) : «faire dessiner le terrain» pour l'ériger en support de médiation entre chercheurs. Ainsi, dès le premier jour, chaque participant a été invité à dessiner sa problématique de recherche, puis à présenter son dessin afin de partager sa posture avec le groupe. Un autre travail de mise en images, collectif cette fois-ci, a été réalisé à partir du matériau réuni durant la visite de terrain. L'idée était de construire collectivement un diaporama puis de le commenter pour l'ensemble du groupe.

Ces choix imposaient un cadre contraignant aux échanges: temps de travail, outils et supports du dialogue, rythme de l'atelier, etc. Toutefois, le canevas laissait à chacun la possibilité de composer dans ces contours, notamment en interprétant les flous laissés dans les consignes.

\section{Partager les points de vue par l'image: un pas vers l'autre, un pas de côté}

Le partage des points de vue sur l'objet s'est d'abord organisé autour de la mise en images des canaux. L'image, qu'il s'agisse de dessins ou de photographies, est un support pertinent pour dialoguer, à travers la mise en visibilité des manières différentes d'aborder la réalité, et pour faciliter l'émergence de nouvelles interprétations d'une situation.

\section{Dessiner sa problématique: une portée explicative}

La mise en récit des dessins individuels était l'occasion pour chacun de rendre explicites ses intérêts. De nombreux éléments graphiques se sont révélés communs (représentation spatialisée des canaux, présence d'eau et d'acteurs). Leur lecture a alors dégagé l'impression d'une vision partagée de l'épaisseur sociale et physique des canaux.

\footnotetext{
${ }^{5}$ B. Bonté, praticien de cette forme d'expression, a accepté d'animer ce temps de l'atelier.
} 


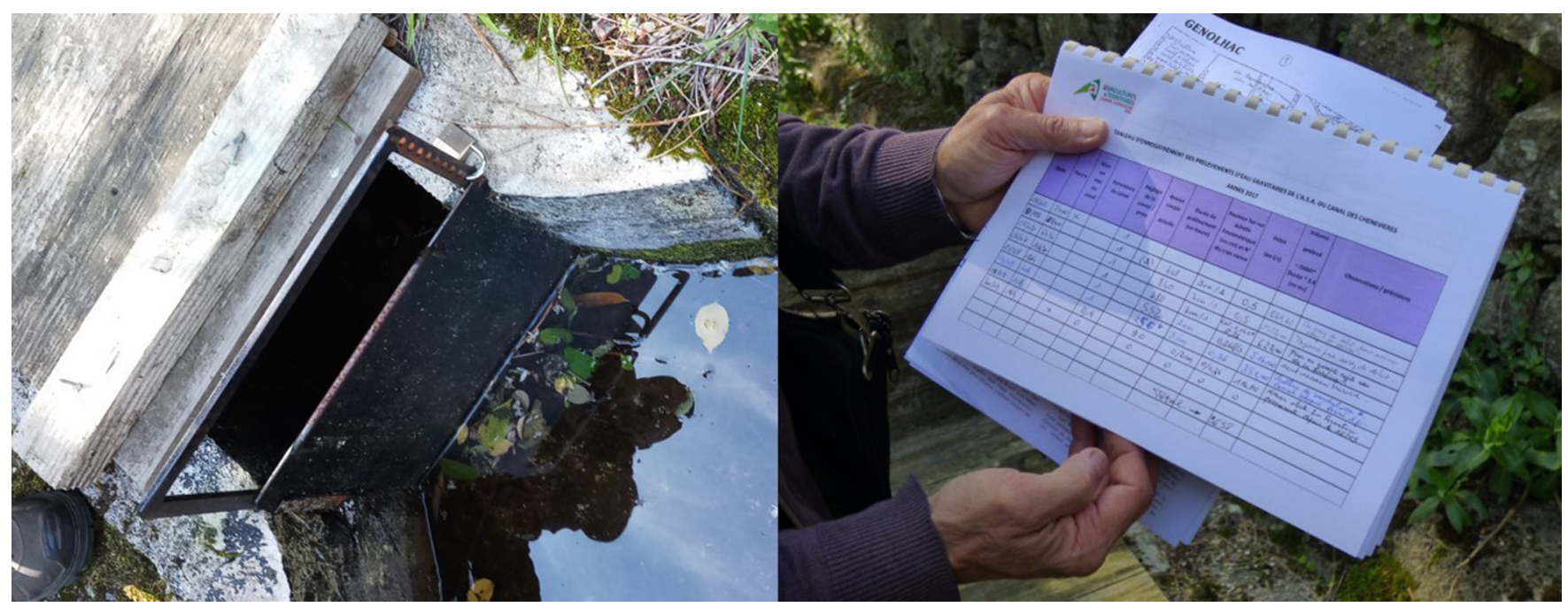

Fig. 2. À gauche, martelière équipée d'une échelle crantée. Son installation découle des mesures de suivi adoptées par l'administration. La position de la vanne permet de réguler le prélèvement. À droite, chaque association d'irrigants doit renseigner les changements de position de la martelière. Clichés : à gauche, N. Kherbache; à droite, T. Ruf.

Cependant, à l'écoute des récits, l'explication des proportions données à un élément ou de sa centralité, par exemple, a fait émerger la variété des approches. La place donnée aux canaux dans la construction des interrogations est particulièrement éclairante. Point de départ de l'élaboration de leur problématique pour certains, ils sont, pour d'autres, un élément de contexte, un « morceau » de l'histoire locale ou encore un facteur susceptible de modifier un paramètre du modèle. Quant à l'inscription territoriale des canaux, certes partagée, elle ne traduit pas toujours des préoccupations communes. Alors que les représentations d'un espace irrigué renvoient aux dynamiques sociales et/ou techniques au sein de cet espace, celles centrées sur un bassin (dé)versant témoignent plutôt du souci d'interroger l'articulation des canaux avec d'autres domaines de la gestion de l'eau. Les modalités d'illustration de la dimension sociale méritent aussi réflexion. Cette dernière est le plus souvent symbolisée par des bonshommes. Mais ils ne renvoient pas tous aux mêmes figures du social, faisant référence à «l'usager» (irrigants, riverains, «préleveurs ») ou recouvrant l'hétérogénéité des acteurs de l'eau (administrations, techniciens de rivière, chercheurs, gestionnaires de canaux). L'illustration des interactions entre acteurs et leur explicitation témoignent aussi de la variété des problématiques de recherche. La mention d'outils de mesure (calculatrice, courbes de débit) traduit pour sa part une analyse de l'action publique. D'autres privilégient l'usage de mots et de flèches pour signaler l'intérêt d'une étude des interactions sociales. Ainsi, les représentations de la matérialité, des échelles spatiales, des acteurs et la manière d'articuler ces éléments permettent de toucher du doigt la diversité d'interprétations de ce que le « social» recouvre. Cela conduit aussi à envisager le risque qu'il y a à considérer qu'en parler, le mentionner ou le dessiner suffise à comprendre ce que chacun met derrière l'idée de «social». Une démonstration identique pourrait être faite autour de l'eau. Figurant dans tous les dessins, sa mise en mots en révèle les différentes dimensions, tour à tour ressource, élément du paysage ou objet hybride ${ }^{6}$.

Les dessins renvoient à l'expérience interdisciplinaire de leurs auteurs. Ainsi, rien de surprenant à ce qu'un participant identifié comme hydrologue représente sa problématique par l'association d'acteurs et de courbes, dans la mesure où il a reçu une formation pluridisciplinaire (agronomie, sciences politiques) qui l'invite à interroger les modalités de couplage des actions humaines et des régimes hydrologiques. Par ailleurs, rien d'étonnant à ce que la sociologue habituée à travailler avec des hydro(géo)logues représente les échanges de flux de surface et souterrains.

\section{Faire valoir ses intérêts dans l'interprétation collective du terrain}

Lors du travail en groupe sur le terrain, les participants ont été conviés par leurs interlocuteurs de Génolhac à suivre les « chemins de l'eau » (Aubriot, 2000), de la prise d'eau aux parcelles. Dans les diaporamas, les dimensions matérielles des béals illustrent une situation, une idée, pour constituer un point de départ à une observation, à une réflexion ou encore à une analogie (Fig. 2).

\footnotetext{
${ }^{6}$ L'analyse des dessins et des explications données par leurs auteurs a été réalisée après l'atelier. Il serait intéressant d'intégrer cette phase du dialogue dans le canevas de l'atelier, peut-être en favorisant une réflexion parallèle côté «sciences de la nature» et côté «sciences de la société».
} 


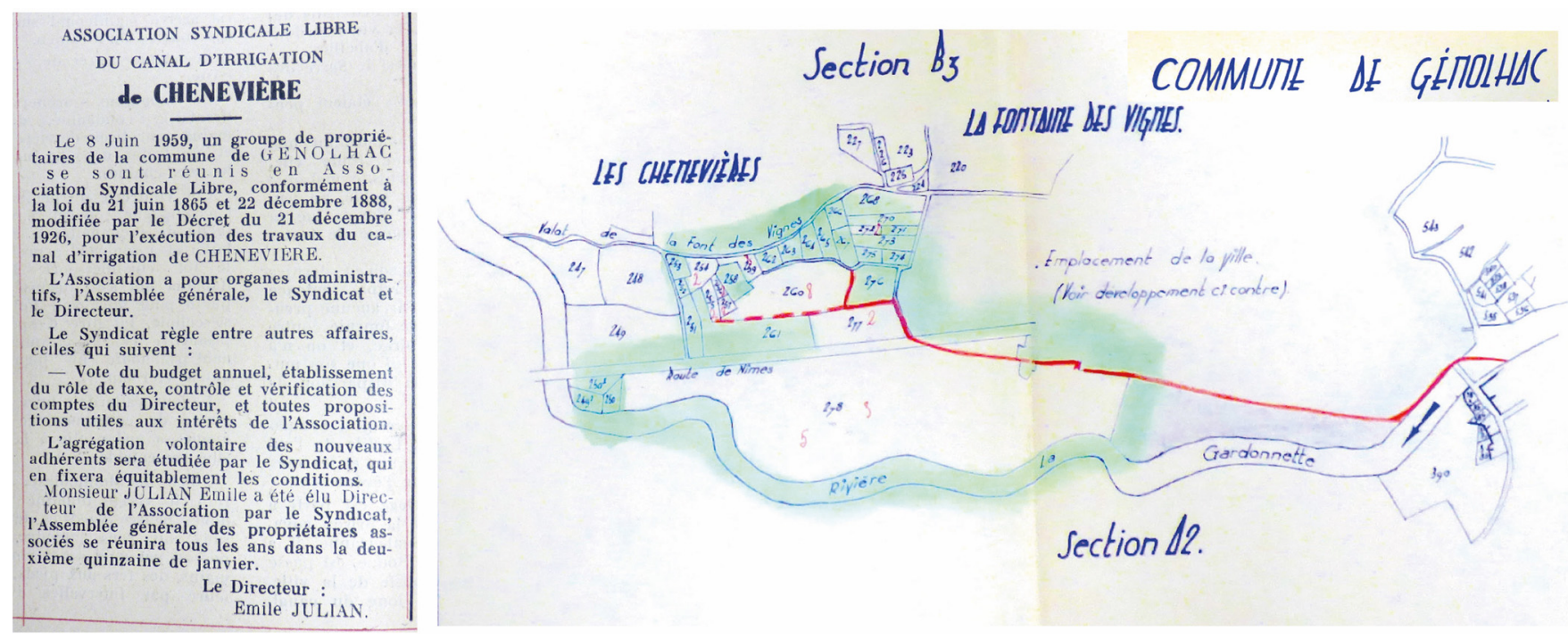

Fig. 3. Des documents d'archives illustrent l'historicité des modalités de partage de l'eau. Source: mairie de Génolhac; cliché : T. Ruf.

La composition des trois diaporamas révèle des interprétations partagées du terrain. Le déclin des béals et le caractère exceptionnel des aménagements sont deux des idées récurrentes de l'atelier. Les premières interprétations sont échangées pour expliquer l'engagement de nos interlocuteurs sur le terrain dans le maintien (physique et institutionnel) des béals. L'historicité de l'ouvrage (Fig. 3) et son ancrage dans l'espace habité sont mentionnés pour évoquer l'importance de la transmission de ce patrimoine. Une attention particulière est également donnée aux travaux de modernisation. Ainsi, plusieurs photographies illustrent les réparations des ouvrages ou le remplacement des canaux par des tuyaux. La discussion s'attarde sur les contraintes réglementaires ; les participants partagent leur étonnement face à une forme d'insistance réglementaire en faveur d'une nouvelle régulation d'ouvrages quasiment abandonnés.

Un intérêt commun autour de la rencontre entre une situation locale et des mesures réglementaires se dessine facilement. Mais l'interprétation collective qu'implique l'exercice de construction d'un diaporama impose des choix. Quels clichés mettre en avant? Sur quels points insister? Dans un des groupes, ce travail s'est organisé à partir d'un aspect important pour l'un des membres qui s'intéresse aux effets des canaux gravitaires et de leur gestion sur l'hydrologie de la rivière. Au fil des discussions, l'importance de la présence des vannes dans ce travail a fait écho aux expériences d'autres membres du groupe. Les réflexions se sont alors centrées sur l'évolution des contraintes réglementaires en les replaçant dans l'histoire institutionnelle des canaux. Dans ce cas, le diaporama apparaît comme le fruit d'une négociation où chacun a assumé et défendu les spécificités de son regard et son interprétation des faits observés. La présentation orale du diaporama par l'un des membres du groupe a aussi impliqué un travail d'appropriation et/ou de traduction des différents lexiques, pour passer d'une discipline à l'autre. Cette étape participe du travail de clarification et de construction interdisciplinaire.

Les exercices de mise en images présentent l'intérêt de délimiter des moments particuliers dans les échanges. Pendant le temps du dessin et de son explication, chacun a présenté sa posture, avec la volonté de rendre explicites ses préoccupations. Comme l'a exprimé l'un des participants, cet exercice a suscité chez lui un pas réflexif sur sa manière d'aborder l'objet commun, dans la mesure où il s'est accompagné d'une déconstruction de l'apparente homogénéité de ses propres représentations, en miroir de celles d'autrui. Ce moment complète bien l'exercice de construction d'une interprétation collective du terrain. En effet, l'association des deux exercices a d'abord permis de constituer un socle de discussion à partir de situations différenciées, puis de mettre en relation des regards individuels sur une même situation, pour enfin réintroduire des éléments importants pour certains, qui ne l'étaient pas a priori pour d'autres.

\section{Glissements de terrain : à la recherche de convergences}

Les temps de discussion collective qui ont suivi le moment dédié au terrain étaient organisés autour de grandes questions transversales, les «glissements de terrain ». Pensées à l'origine pour permettre l'expression de divergences, donc favoriser la «négociation» interdisciplinaire, ces grandes questions ont été saisies par les participants pour souligner les convergences entre terrains, époques et disciplines. 


\section{Du Sud au Nord, du Nord au Sud: rapprochement des expériences de terrain}

Le premier «glissement» visait le dépassement des dichotomies classiquement effectuées entre situations du Nord et du Sud. Dans cette discussion, les spécificités de chacun ont été mises de côté pour laisser place à une volonté partagée d'établir des rapprochements entre terrains. Dans son intervention, Stéphane Ghiotti, sollicité comme témoin pour ce "glissement», a livré un récit de sa propre expérience de terrain qui l'a mené de la France au Liban. Il a insisté en particulier sur la manière dont l'étude de situations a priori très éloignées finit par donner du sens à l'objet étudié. Ce récit a fait émerger d'autres rapprochements, notamment la mise en perspective des enjeux de l'eau sur un terrain français au regard des formes d'hybridation des règles de gestion observées en Algérie. La question du devenir de l'irrigation gravitaire s'est présentée comme un liant entre terrains des uns et des autres. Ainsi, par exemple, les béals de Génolhac sont en quelque sorte placés «au bout de l'histoire» d'un déclin dont les différents stades sont encore observables au Cambodge.

La recherche de commun s'est également traduite par des glissements de vocabulaire. La dimension matérielle des béals rappelle celle de tout système irrigué par canaux gravitaires. De cette façon, le béal se fait tour à tour prek, seguia ou targa selon les terrains évoqués, le répartiteur fait penser à la kesria, "peigne» utilisé en contexte oasien pour la répartition de l'eau. La dimension sociale sous-tendue par cette matérialité est aussi évoquée. Ainsi, la journée d'entretien des béals est-elle comparée à la twiza marocaine, forme de travail collectif. Si certains avaient déjà établi des ponts entre des cas très contrastés, ce glissement de terrain a été l'occasion pour d'autres de s'étonner de la singularité de leur propre terrain, mais aussi de leur étonnante ressemblance avec des situations très éloignées. Comme l'exprime l'un des participants, il est intéressant «d'aller voir le terrain de l'autre [...] car cela permet de penser, de réfléchir le sien différemment ». Ici, le terrain comme catalyseur n'est pas une pratique partagée, il est une expérience individuelle dans laquelle chacun puise des éléments de dialogue et de croisement des regards.

\section{Discuter des temporalités: temps de la recherche, temps des canaux}

Le deuxième "glissement» était celui de la temporalité des questions de recherche posées autour des canaux d'irrigation communautaires. L'attente des organisateurs était de croiser les expériences sur cet objet pour interroger ce qui fait et défait nos postures scientifiques. Mais cette orientation n'a pas fait sens pour tous et la question de la temporalité a été appropriée selon deux angles distincts : le temps de la recherche et celui des canaux.

Le « temps de la recherche» a été abordé pour resituer l'intérêt et l'émergence des travaux sur l'irrigation communautaire. Le contexte international des années 1980 est rappelé à travers l'évocation des thèses de Hardin (1968) et de Ostrom (1992) qui ont mis sur le devant de la scène des problématiques de recherche déjà anciennes. Des travaux d'anthropologues ou d'historiens sont mentionnés pour rappeler cette antériorité. Les recherches sur l'irrigation gravitaire menées par les collègues présents sont alors affichées comme une manière de contribuer à penser la capacité des sociétés locales à gérer l'eau en dehors d'interventions publiques centralisées, cela au risque d'une certaine idéalisation de l'irrigation "communautaire " comme tient à le souligner l'un des participants. Ces travaux sont engagés, rejoignant la posture des participants qui ancrent leur recherche comme une déconstruction du «mythe» de la toute-puissance de la grande hydraulique d'État ou dans une critique du discours des organismes internationaux. La reconnaissance des apports des sciences sociales sur les questions relatives à l'eau est soulignée comme s'inscrivant dans les «temps de la recherche». Elle est interprétée comme génératrice du renouvellement des questions scientifiques, les disciplines des sciences humaines et sociales se saisissant autrement de l'irrigation communautaire pour interroger la matérialité des rapports sociaux autour de l'eau (Aubriot, 2013) ou les rapports entre sociétés et environnement (Aspe, 2012). Ces avancées permettent de renouveler et d'enrichir l'analyse des règles et du partage de l'eau. Par ailleurs, la recomposition des communautés de chercheurs incite au croisement des regards disciplinaires (Leblan, 2016). À ce titre, l'exemple des foggaras en Algérie est éclairant. L'un des participants rapporte un discours, principalement issu des sciences du physique, selon lequel il n'y a plus rien à dire sur ces objets «archaïques». Or, l'association de la géographie sociale et de la modélisation d'acteurs a permis de porter un regard neuf sur les foggaras, en montrant leur rôle central dans la réorganisation de l'espace physique et social, au vu des transformations de l'agriculture de la région (Idda, 2019). On retrouve une volonté partagée de contribuer à la production d'un discours alternatif sur l'irrigation communautaire et ses instruments techniques.

La discussion sur «les temps des canaux» a été introduite par Thierry Ruf, sollicité comme témoin sur ce «glissement». Il propose l'idée de cycles hydroinstitutionnels pour prendre en compte des dynamiques de long terme dans la compréhension des mutations observées autour de l'irrigation communautaire. Ces perspectives éclairent les interrogations du groupe sur la «modernisation» des béals de Génolhac, comme l'illustre l'exemple des martelières. Alors qu'elles ont 
été conçues à l'origine pour laisser passer un débit suffisamment important pour assurer le fonctionnement de moulins, le débit restreint par les réglementations contemporaines impose une modification drastique de ces ouvrages. De fait, les martelières ne laissent désormais passer qu'un filet d'eau parfois insuffisant pour assurer le fonctionnement hydraulique du béal. Cette vanne symbolise ainsi l'obsolescence d'une forme ancienne d'organisation sociotechnique autour de l'eau. Il est intéressant de noter que l'entrée par le «temps des canaux » révèle là aussi une posture partagée par les participants. Sans pour autant être formulée explicitement, une critique de la "panacée technique» productrice de normes à l'épreuve du temps semble faire consensus.

\section{D'une discipline à l'autre, une réflexivité inconfortable}

Échanger autour des implications du croisement des regards était le troisième glissement qui composait le canevas de l'atelier. Jeanne Riaux, positionnée en témoin sur ce thème, a observé que le dialogue interdisciplinaire avait bénéficié d'une double entrée: matérialité et terrain. Quelles que soient leurs disciplines scientifiques, tous les participants ont en effet suivi le tracé du canal pour le restituer en diaporama. Le choix de l'objet hydraulique "canaux communautaires » a été déterminant en cela, faisant plus appel aux expériences préalables de terrain qu'aux ancrages disciplinaires. De ces lectures d'un réseau d'irrigation, les manières dont chacun se figure l'objet remontent à la surface : quel est le débit entrant? Qui sont les ayants droit? Ya-t-il des dérivations illicites? etc. Ces questions organisent le schéma de représentation de l'objet porté par chacun et permettent des échanges entre participants. Ainsi le dialogue s'est-il engagé autour de questions comme l'entretien des réseaux et le calibrage des ouvrages à partir d'interrogations sur l'existence de droits formels et informels.

Les participants ont exprimé un réel plaisir à partager ce temps collectif et à découvrir la manière dont chacun construit sa vision des canaux. Les échanges se sont déroulés dans une volonté d'écoute mutuelle qui a favorisé certains apprentissages. Toutefois, le moment d'écriture dont procède cette réflexion a permis de révéler quelques points de divergences, à peine esquissés dans les discussions. Ainsi, par exemple, le rapport aux disciplines mis en avant dans l'organisation de l'atelier est-il interrogé par des participants qui ne s'identifient pas à une seule discipline, voire à plusieurs. C'est tout particulièrement le cas pour ceux qui font de la modélisation dont la pratique consiste à intégrer des concepts de disciplines différentes. La manière d'interpréter certaines de leurs postures a dû être révisée, en essayant d'élargir le point de vue grâce aux critiques exprimées. Dans cette perspective, l'appropriation du « glissement» autour des temporalités ouvre des voies de dialogue intéressantes pour approfondir le travail réflexif sur les postures individuelles. Cette appropriation a effectivement semblé fédérer une posture commune d'engagement, créant du commun entre les participants. Pour autant, le consensus esquissé ne signifie pas qu'il y ait une vision partagée de ce que comporte cet engagement. La prise de conscience et l'explicitation des regards singuliers permettraient d'approfondir les débats d'idées et de postures.

Un autre aspect autour duquel aurait pu s'engager la négociation interdisciplinaire est celui de la «continuité écologique» pour aborder le devenir des canaux communautaires. Cette notion est centrale pour plusieurs des participants qui l'ont régulièrement invoquée au cours de l'atelier, débattant même de sa problématisation. L'explicitation de cet intérêt a permis à certains qui n'y prêtaient pas attention de s'en saisir pour poser des questions de performance hydraulique, de construction de normes ou de rapports à la nature. Ainsi une partie du groupe a-t-elle progressivement fait valoir l'intérêt de considérer la notion de «continuité écologique» pour s'intéresser au devenir des canaux. C'est là un exemple encourageant de négociation porteuse de nouvelles questions de recherche.

Un autre constat formulé par les participants est celui de s'être sentis libres de s'exprimer dans l'espace ouvert par les "glissements de terrain», alors que d'autres moments du dialogue ont pu susciter une forme d'inconfort ou de "déstabilisation organisée». Mais, cet aspect du dialogue n'a pas été mis à profit pour orienter les échanges. L'écriture de ce texte est alors entendue comme un moment privilégié pour poursuivre la réflexion sur ce sentiment d'inconfort et de déstabilisation que peut provoquer le croisement des regards. Dans un ouvrage, Becker (2004) raconte le malaise de ses étudiants lorsqu'il leur demande de soumettre à la critique de leurs camarades un texte non abouti. Tous sont récalcitrants à livrer une version non conceptualisée de leurs idées, facilement critiquable en l'absence d'argumentation théorique solide. Le travail réflexif auquel invite l'interdisciplinarité peut susciter une telle réaction de prudence ou de pudeur. En effet, l'acte de croiser son regard avec celui d'autrui implique de dévoiler ses propres incertitudes et contradictions. Ce type de dialogue renvoie aussi à sa «communauté épistémique » et à sa capacité d'en discuter les positions. Mais la déstabilisation peut aussi être considérée comme inhérente au processus de négociation, rappelant à chacun ses propres limites vis-à-vis de sa discipline, par exemple en matière de maîtrise des fondamentaux ou de capacité à argumenter une position dans sa "communauté ». Le fait d'admettre ces limites permettrait peut-être 
de se défaire d'une posture disciplinée et/ou d'une tentation d'imposer le point de vue de sa discipline pour entrer dans la négociation.

\section{Un canevas original pour le dialogue interdisciplinaire}

L'atelier sur les canaux communautaires a permis de tester des moyens d'engager la pratique interdisciplinaire et d'y réfléchir. Le canevas proposé se présente alors comme un « résultat» de la progression du dialogue. Les interrogations qui ont motivé l'organisation de cet atelier s'inscrivent dans un dispositif plus large que nourrissent les membres de l'équipe SocioHydro. À cet égard, l'organisation et le déroulé des rencontres autour des canaux ont été riches d'enseignements, l'écriture de ce texte contribuant à les identifier et à les prolonger par un travail de réflexivité collective. Tout d'abord, l'objet «canaux communautaires» s'est révélé être un objet « passeur de frontières » (Jollivet, 1992) particulièrement riche. Ensuite, le pari de proposer des réflexions par analogie a donné lieu à des rapprochements, permettant de dépasser les postures disciplinaires et les ancrages empiriques, pour déplacer le dialogue vers ce qui fait sens collectivement. En couplant une expérience courte de terrain et la référence à un objet technique partagé, le canevas a répondu à l'une des préoccupations énoncées en introduction, à savoir la possibilité de dialoguer en l'absence d'une expérience commune de terrain sur le temps long.

Un autre pari réussi est celui d'avoir proposé aux participants un moment de respiration dans un contexte où la recherche s'accélère (Stengers, 2013). Cependant, la bonne entente recherchée a parfois été source de frustrations. Certains échanges sont restés cantonnés à une mise en commun polie ne permettant pas le dévoilement de contradictions entre les approches en présence. Pour les organisateurs, la déstabilisation n'a pas été suffisamment loin, ce qui confirme la difficulté de construire un dialogue interdisciplinaire. Cependant, ces frustrations ne sont pas partagées par l'ensemble des participants. Ceux qui ne s'identifient pas à une posture disciplinaire dominante semblent considérer que la prise de connaissance des approches d'autrui nourrit leur représentation du réel et constitue un résultat en soi. La réflexivité proposée a été bien accueillie car elle est assez nouvelle dans leur pratique de l'interdisciplinarité.

Par ailleurs, la relecture de ce texte par les participants de l'atelier a mis en avant une autre difficulté du dialogue interdisciplinaire qui dépasse les disciplines, pour concerner les manières de concevoir l'interdisciplinarité. Cette difficulté renvoie à la nature même de ce dialogue. Établir un dialogue avec des personnes porteuses de définitions multiples de l'interdisciplinarité serait-il une voie à suivre pour concevoir d'autres manières de faire? Dans cette perspective, interroger la composition du groupe réuni prend tout son sens. Est-ce que la constitution d'une forme d'entre-soi, à force de rencontres, favoriserait un dialogue où chacun se sentirait à sa place et trouverait un intérêt à approfondir les débats? Une démarche de coconstruction du canevas pourrait alors servir de médiateur. Ou bien, au contraire, faut-il solliciter des chercheurs «disciplinés» qui par leurs interrogations participeraient à un dialogue construit autour et à partir de regards disciplinaires divergents?

Il faut aussi prendre en compte la possibilité que les frustrations soient partie intégrante du processus de dialogue. Considérées comme moteur de la réflexivité, voire un signe d'engagement dans le dispositif interdisciplinaire, ne seraient-elles pas propices à la déstabilisation? Faut-il alors imaginer les nourrir ou au contraire les réduire pour éviter qu'à force de répétitions elles n'essoufflent celui qui les vit? Et pour cela, faut-il resserrer encore un peu plus les échanges autour d'un canevas très structuré ou bien au contraire élargir l'espace de liberté ? Ces interrogations illustrent combien l'interdisciplinarité se présente comme une progression collective, mais peut-être surtout comme un moyen d'ouvrir le dialogue entre chercheurs et de donner du sens à nos entreprises scientifiques individuelles.

\section{Remerciements}

Les auteurs remercient les participants pour leur engagement dans la réalisation de cet atelier et dans la rédaction de cette réflexion. Nous remercions également l'IRD, INRAE et l'Agence de l'eau Rhône-Méditerranée-Corse dans le cadre de l'accord avec la Zone atelier Bassin du Rhône (ZABR) pour avoir participé au financement de l'atelier.

\section{Références}

Araújo E., Guimarães B., 2018. The future is but a word. Exploring the scientist's discourses on the future and their implications, Temporalités. Revue de sciences sociales et humaines, 28, https://doi.org/10.4000/temporalites.5546.

Arnauld de Sartre X., Petit O., 2018. Une nouvelle géographie de l'enseignement supérieur et de la recherche: rapprochements, concentrations, tensions... et opportunités? Nature Sciences Sociétés, 26, 3, 255-256, https://doi.org/ $10.1051 / \mathrm{nss} / 2018049$

Aspe C. (Ed.). 2012. De l'eau agricole à l'eau environnementale. Résistance et adaptation aux nouveaux enjeux de partage de l'eau en Méditerranée, Versailles, Éditions Quæ.

Aubriot O., 2000. Comment «lire» un système d'irrigation? Une approche pour l'étude de systèmes irrigués tradition- 
nels, illustrée de cas pris au Népal, Territoires en mutation, 7, 37-50.

Aubriot O., 2013. De la matérialité de l'irrigation. Réflexions sur l'approche de recherche utilisée, Journal des anthropologues, 132-133, 1-2, 123-144, https://doi.org/10.4000/ jda. 4931 .

Becker H.S., 2004. Écrire les sciences sociales. Commencer et terminer son article, sa thèse ou son livre, Paris, Economica.

Bühler È.-A., Cavaillé F., Gambino M., 2006. Le jeune chercheur et l'interdisciplinarité en sciences sociales. Des pratiques remises en question, Natures Sciences Sociétés, 14, 4, 392-398, https://doi.org/10.1051/nss:2007007.

Calandra M., 2013. Faire dessiner le terrain. La nature à « risques » et les jardins de subsistance de Tanna et Tongoa (Vanuatu), Techniques \& Culture, 60, 182-201, https://doi. org/10.4000/tc.6822.

Ghiotti S., 2007. Les territoires de l'eau. Gestion et développement en France, Paris, CNRS Éditions.

Hardin G., 1968. The tragedy of the commons, Science, 162, 3859, 1243-1248, https://doi.org/10.1126/science.162.3859.1243.

Hmouri A., Bouzidi Z., Kuper M., 2017. Révéler ce qui fait communauté dans un système irrigué par l'analyse des dynamiques conflictuelles, Revue marocaine des sciences agronomiques et vétérinaires, 5, 1, 83-95, https://www. agrimaroc.org/index.php/Actes_IAVH2/article/view/472.

Idda S., 2019. Aménagement de l'espace oasien à foggara face aux changements des conditions hydrogéologiques et socioéconomiques. Thèse de doctorat, Oran, Université d'Oran.

Ingold A., 2008. Les sociétés d'irrigation: bien commun et action collective, Entreprises et histoire, 50, 1, 19-35, https://doi.org/10.3917/eh.050.0019.

Jollivet M. (Ed.), 1992. Sciences de la nature, sciences de la société. Les passeurs de frontière, Paris, CNRS Éditions, https://doi.org/10.4000/books.editionscnrs.4154.

Jollivet M., Legay J.-M., 2005. Canevas pour une réflexion sur une interdisciplinarité entre sciences de la nature et sciences sociales, Natures Sciences Sociétés, 13, 2, 184-188, https:// doi.org/10.1051/nss:2005030.

Latour B., 2013. Nous n'avons jamais été modernes. Essai d'anthropologie symétrique, Paris, La Découverte.

Leblan V., 2016. Les disciplines mènent à tout à condition d'en sortir, in Hubert B., Mathieu N. (Eds), Interdisciplinarités entre Natures et Sociétés, Bruxelles, Peter Lang, 207-223.
Massuel S., Riaux J., Molle F., Kuper M., Ogilvie A., Collard A.-L., Leduc C., Barreteau O., 2018. Inspiring a broader socio-hydrological negotiation approach with interdisciplinary field-based experience, Water Resources Research, 54, 4, 2510-2522, https://doi.org/10.1002/2017WR021691.

Mathieu N., 2012. L'interdisciplinarité entre natures et sociétés, vingt ans après : le point de vue d'une géographe engagée, Écologie politique, 45, 2, 71-81, https://doi.org/ 10.3917/ecopo.045.0071.

Mathieu N., Rivault C., Blanc N., Cloarec A., 1997. Le dialogue interdisciplinaire mis à l'épreuve: réflexions à partir d'une recherche sur les blattes urbaines, Natures Sciences Sociétés, 5, 1, 18-30, https://doi.org/10.1051/nss/ 19970501018.

Ostrom E., 1992. Crafting institutions for self-governing irrigation systems, San Francisco, ICS Press.

Pivot A., Mathieu N., 2007. Agnès Pivot : l'écriture, processus heuristique et outil pour le dialogue dans une démarche interdisciplinaire. Entretien, Natures Sciences Sociétés, 15, 4, 411-416, https://doi.org/10.1051/nss:2008011.

Riaux J., 2013. Engager la construction d'un regard sociohydrologique: des archives catalyseurs de l'interdisciplinarité, Natures Sciences Sociétés, 21, 1, 15-23, https:// doi.org/10.1051/nss/2013061.

Riaux J., Massuel S., 2014. Construire un regard sociohydrologique (2). Le terrain en commun, générateur de convergences scientifiques, Natures Sciences Sociétés, 22, 4, 329-339, https://doi.org/10.1051/nss/2014046.

Rivière-Honegger A., Ruf T. (Eds), 2000. Approches sociales de l'irrigation et de la gestion collective de l'eau : démarches et expériences en France et dans le monde, Territoires en mutation, 12.

Stengers I., 2013. Au temps des catastrophes. Résister à la barbarie qui vient, Paris, La Découverte.

Valat de Chapelain G., 1983. Histoire économique sociale et culturelle des vallées de la Cèze, perçue à partir de l'utilisation de l'énergie hydraulique. Mémoire de diplôme d'études approfondies en histoire et civilisation, Aix-enProvence, Université d'Aix-en-Provence.

Wateau F., 2016. On ne badine pas avec le progrès. Barrage et village déplacé au Portugal, Paris, Éditions Fondation Maison des sciences de l'homme.

Citation de l'article : Collard A.-L., Riaux J., Massuel S., Bonté B., Laurenceau M., Richard B., Rivière-Honegger A. Construire un regard sociohydrologique (3). Expérimenter un canevas pour engager la négociation interdisciplinaire. Nat. Sci. Soc. 29, 1, 77-86. 